\title{
Molecular Mechanisms of AIPL1 and its TPR domain in Leber congenital amaurosis 4 (LCA4) a severe form of childhood blindness.
}

\author{
Ravi Prakash Yadav ${ }^{1}$, Lokesh Ghakar ${ }^{2}$, Liping Yu², Nikolai O. Artemyev ${ }^{1}$ \\ ${ }^{1}$ Department of Molecular Physiology and Biophysics, University of lowa, lowa City, IA \\ ${ }^{2}$ Department of Biochemistry, University of lowa, lowa City, IA
}

Aryl hydrocarbon receptor-interacting protein-like 1 (AIPL1) is a specialized chaperone of the visual effector enzyme phosphodiesterase-6 (PDE6). Mutations in AIPL1 cause Leber congenital amaurosis type 4 (LCA4), a severe form of childhood blindness. However, the structure-function relationships and the mechanisms of LCA-causing AIPL1 mutations are poorly understood. AIPL1 is comprised of an FK506-binding protein (FKBP) domain and a tetratricopeptide (TPR) domain. The interaction of AIPL1 FKBP domain (AIPL1-FKBP) with the isoprenyl groups on PDE6 is essential to the chaperone function. We have solved the crystal structure of the AIPL1 FKBP domain and revealed the molecular basis of the prenyl lipid binding to this domain. We demonstrated that several LCA-linked mutations in the FKBP-domain disrupt this interaction. Furthermore, we have shown that AIPL1 interacts with the regulatory Py subunit of PDE6 and recently, we identified a novel interaction between the AIPL1 TPR domain and the regulatory PY-subunit of PDE6. This interaction is critical for the chaperone function of AIPL1, since $\mathrm{PY}$ is found to serve as a potent co-chaperone with AIPL1 in the folding and assembly of PDE6. We hypothesize that the AIPL1/ Py interaction underlies the ability of Py to act as a potent co-chaperone of PDE6. To delineate the interactions and the role of AIPL1-TPR, we solved its crystal structures in the apo form. Currently, we are mapping Py binding site on AIPL1-TPR domain by NMR, fluorescence, and biolayer interferometry assays. Our preliminary data suggest that Py may acts as an affinity adaptor for AIPL1 by interacting with the TPR domain, thereby greatly enhancing the chaperone activity and selectivity of AIPL1 towards its client. 